\title{
Interactive evolutionary multiple objective optimization algorithm using a fast calculation of holistic acceptabilities e-Appendix (supplementary material)
}

\author{
Michał K. Tomczyk, Miłosz Kadziński \\ Institute of Computing Science, Poznań University of Technology \\ Poznań, Poland \\ \{michal.tomczyk,milosz.kadzinski\}@cs.put.poznan.pl
}

ACM Reference Format:

Michał K. Tomczyk, Miłosz Kadziński. 2021. Interactive evolutionary multiple objective optimization algorithm using a fast calculation of holistic acceptabilities e-Appendix (supplementary material). In Genetic and Evolutionary Computation Conference (GECCO '21), Fuly 10-14, 2021, Lille, France. ACM, New York, NY, USA, 6 pages. https://doi.org/10.1145/3449639.3459294

In the e-Appendix, we discuss the material which was not included in the main paper for the sake of conciseness. Specifically:

(1) we discuss the results attained by iMOEA-HA coupled with different selection procedures SEL (Section 1);

(2) we present the results attained by iMOEA-HA parameterized with different queue limits $K$ (Section 2);

(3) we compare the execution times of EMOSOR and iMOEA-HA coupled with different queue limits $K$ (Section 3 );

(4) we compare different algorithms applied to the WFG1 benchmark problem (Section 4).

The experimental results provided in this e-Appendix follow the evaluation strategy discussed in the main paper.

\section{COMPARISON OF DIFFERENT VARIANTS OF IMOEA-HA COUPLED WITH VARIOUS SELECTION PROCEDURES}

In this section, we verify the performance of iMOEA-HA coupled with two selection procedures $S E L$ :

- $S E L=T U R$ : Parent solutions are selected using a tournament of size 2. During the selection process, a solution included in a more favorable non-dominated front is promoted. In case of a tie, a solution with a lesser $H A-I N V$ score is preferred.

- $S E L=R M P$ : The mating pool is restricted by randomly selecting parent solutions from a subset of non-dominated solutions assigned to a random queue.

We ran the algorithms for WFG1-9 and $M=2, \ldots, 5$. Furthermore, the analysis was performed for different queue limits $K \in$ $\{5,10,15,20\}$. The results of this comparative study are provided in Tables $1(K=5), 2(K=10), 3(K=15)$, and $4(K=20)$.

Permission to make digital or hard copies of all or part of this work for personal or classroom use is granted without fee provided that copies are not made or distributed for profit or commercial advantage and that copies bear this notice and the full citation on the first page. Copyrights for components of this work owned by others than ACM must be honored. Abstracting with credit is permitted. To copy otherwise, or republish, to post on servers or to redistribute to lists, requires prior specific permission and/or a fee. Request permissions from permissions@acm.org.

GECCO '21, fuly 10-14, 2021, Lille, France

(C) 2021 Association for Computing Machinery.

ACM ISBN 978-1-4503-8350-9/21/07 . \$15.00

https://doi.org/10.1145/3449639.3459294
The results indicate that, in general, both variants of iMOEA-HA perform similarly. However, apart from some individual scenarios, iMOEA-HA restricting the mating pool $(S E L=R M P)$ reported better results for WFG1-2, different numbers of objectives and queue sizes. For instance, for WFG1 and $K=10$ or 15, iMOEA-HA with $S E L=R M P$ outperformed its $S E L=T U R$ counterpart for all numbers of objectives. When it comes to WFG2, the former method proved a better performer when coupled with $K \in\{5,10,15,20\}$ and run for $M=2$. Note that WFG1-2 problems have particularly challenging shapes of PFs. Therefore, we conclude that restricting the mating pool increased the chances for generating better offspring in such complex fitness landscapes.

\section{COMPARISON OF DIFFERENT VARIANTS OF IMOEA-HA PARAMETERIZED WITH VARIOUS QUEUE LIMITS}

This section compares the results attained by iMOEA-HA when coupled with different queue sizes $K$. We consider $K \in\{5,10,15,20\}$, similarly to the main paper. The results of this extensive experiment are provided in Table 5 . They reveal a general tendency that the performance of iMOEA-HA improves with the increase of K. For instance, for WFG1 with $M=5$, the algorithm attained the average ranks $\bar{R}$ of $3.08,2.51,2.33$, and 2.08 for the considered subsequent values of $\mathrm{K}$. The reason is that the greater $\mathrm{K}$ is, the more accurate are the approximations of HA-INV scores used to rank solutions in the population. Hence, the algorithm performs better in predicting the true DM's aspirations, converging the population towards more relevant solutions in the PF.

\section{COMPARISON OF EXECUTION TIMES OF EMOSOR AND IMOEA-HA COUPLED WITH DIFFERENT QUEUE LIMITS}

In this section, we compare the execution times of EMOSOR and iMOEA-HA run for different queue limits K (see Table 6). As discussed in the main paper, iMOEA-HA required less time to perform the simulations. Even for the extreme case, i.e., $K=20$, iMOEA-HA performed, on average, 2.34 faster than EMOSOR. Furthermore, the difference between the computational times of iMOEA-HA and its competitor increases for a greater number of objectives. A more favorable computational complexity of the proposed algorithm derives from imposing a limit for the number of the most preferred ranks considered when calculating the HA-INV scores and using better-suited sorting procedures. 
Table 1: Average ARSD and respective ranks $\bar{R}$ attained by iMOEA-HA with $S E L \in\{T U R, R M P\}$ and $K=5$, applied to WFG1-9 problems with $M=2-5$ objectives.

\begin{tabular}{|c|c|c|c|c|c|c|c|c|c|c|c|c|c|}
\hline & & \multicolumn{3}{|c|}{$M=2$} & \multicolumn{3}{|c|}{$M=3$} & \multicolumn{3}{|c|}{$M=4$} & \multicolumn{3}{|c|}{$M=5$} \\
\hline & SEL & Mean & StD & $\bar{R}$ & Mean & StD & $\bar{R}$ & Mean & StD & $\bar{R}$ & Mean & StD & $\bar{R}$ \\
\hline \multirow{2}{*}{ WFG1 } & TUR & 2.143 & 18.585 & 1.51 & 1.252 & 8.896 & 1.78 & 1.590 & 5.684 & 1.69 & 2.697 & 4.363 & 1.46 \\
\hline & $R M P$ & 1.780 & 15.422 & 1.49 & 0.937 & 6.621 & 1.22 & 1.501 & 5.269 & 1.31 & 2.518 & 3.892 & 1.54 \\
\hline \multirow{2}{*}{ WFG2 } & TUR & 0.639 & 2.434 & 1.76 & 2.219 & 6.615 & 1.68 & 3.174 & 12.726 & 1.52 & 7.228 & 25.564 & 1.47 \\
\hline & $R M P$ & 0.383 & 2.294 & 1.24 & 2.024 & 8.376 & 1.32 & 2.653 & 6.685 & 1.48 & 6.138 & 16.087 & 1.53 \\
\hline \multirow{2}{*}{ WFG3 } & TUR & 0.298 & 2.714 & 1.72 & 0.123 & 0.876 & 1.51 & 0.105 & 0.145 & 1.41 & 0.198 & 0.255 & 1.48 \\
\hline & $R M P$ & 0.601 & 5.700 & 1.28 & 0.157 & 1.036 & 1.49 & 0.106 & 0.156 & 1.59 & 0.190 & 0.177 & 1.52 \\
\hline \multirow{2}{*}{ WFG4 } & TUR & 8.261 & 82.302 & 1.49 & 0.528 & 3.258 & 1.51 & 1.248 & 3.590 & 1.48 & 3.057 & 6.958 & 1.53 \\
\hline & $R M P$ & 0.232 & 1.761 & 1.51 & 0.993 & 8.420 & 1.49 & 1.244 & 2.601 & 1.52 & 2.944 & 5.601 & 1.47 \\
\hline \multirow{2}{*}{ WFG5 } & TUR & 5.747 & 52.899 & 1.48 & 1.283 & 9.926 & 1.53 & 2.145 & 9.106 & 1.62 & 3.823 & 9.082 & 1.51 \\
\hline & $R M P$ & 2.830 & 25.780 & 1.52 & 2.173 & 18.686 & 1.47 & 1.749 & 6.532 & 1.38 & 3.996 & 13.665 & 1.49 \\
\hline \multirow{2}{*}{ WFG6 } & $T U R$ & 2.069 & 17.953 & 1.61 & 1.085 & 7.756 & 1.46 & 1.617 & 3.902 & 1.58 & 2.822 & 4.660 & 1.53 \\
\hline & $R M P$ & 0.918 & 8.501 & 1.39 & 0.878 & 5.719 & 1.54 & 2.750 & 13.900 & 1.42 & 3.047 & 6.989 & 1.47 \\
\hline \multirow{2}{*}{ WFG7 } & TUR & 0.059 & 0.512 & 1.45 & 0.301 & 1.003 & 1.52 & 1.116 & 3.281 & 1.46 & 2.812 & 7.128 & 1.47 \\
\hline & $R M P$ & 0.138 & 1.300 & 1.55 & 0.811 & 6.098 & 1.48 & 1.710 & 6.539 & 1.54 & 3.433 & 8.894 & 1.53 \\
\hline \multirow{2}{*}{ WFG8 } & TUR & 12.001 & 107.595 & 1.51 & 1.888 & 12.983 & 1.58 & 2.359 & 9.412 & 1.68 & 5.011 & 19.609 & 1.54 \\
\hline & $R M P$ & 1.394 & 12.027 & 1.49 & 2.962 & 26.051 & 1.42 & 1.454 & 4.949 & 1.32 & 4.278 & 12.458 & 1.46 \\
\hline \multirow{2}{*}{ WFG9 } & $T U R$ & 2.997 & 21.673 & 1.26 & 1.034 & 6.871 & 1.45 & 2.339 & 9.419 & 1.45 & 4.090 & 9.776 & 1.48 \\
\hline & $R M P$ & 1.991 & 16.818 & 1.74 & 1.841 & 13.174 & 1.55 & 2.264 & 9.001 & 1.55 & 4.221 & 12.490 & 1.52 \\
\hline
\end{tabular}

Table 2: Average ARSD and respective ranks $\bar{R}$ attained by iMOEA-HA with $S E L \in\{T U R, R M P\}$ and $K=10$, applied to WFG1-9 problems with $M=2-5$ objectives.

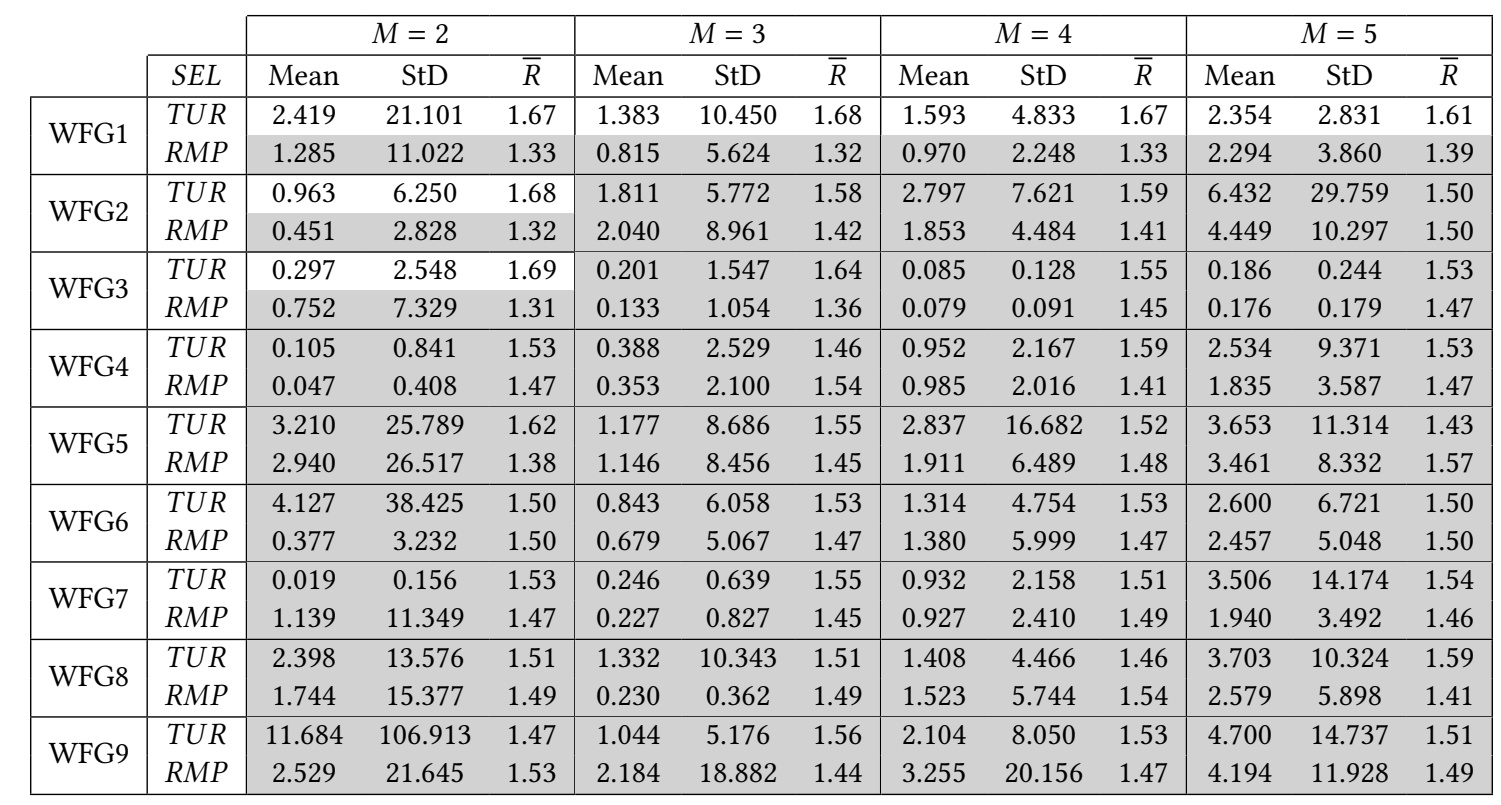

\section{PERFORMANCE OF DIFFERENT ALGORITHMS APPLIED TO THE WFG1 TEST PROBLEM}

This section compares EMOSOR with iMOEA-HA using different selection procedures when applied to the WFG1 test problem. The queue limit was set to $K=15$ for iMOEA-HA. The results of this experiment are provided in Table 7 . They reveal that using the restricting mating pool $(S E L=R M P)$ in iMOEA-HA significantly improved the algorithm's performance compared to the remaining methods. Precisely, iMOEA-HA employing the tournament selection $(S E L=T U R)$ attained the worst average ARSD-based ranks ranging from 2.14 to 2.23 . Therefore, it performed worse than EMOSOR, for which the respective ranks range from 1.86 
Table 3: Average ARSD and respective ranks $\bar{R}$ attained by iMOEA-HA with $S E L \in\{T U R, R M P\}$ and $K=15$, applied to WFG1-9 problems with $M=2-5$ objectives.

\begin{tabular}{|l|c|ccc|ccc|ccc|ccc|}
\multicolumn{1}{c|}{} & \multicolumn{3}{c|}{$M=2$} & \multicolumn{3}{c|}{$M=3$} & \multicolumn{3}{c|}{$M=4$} & \multicolumn{3}{c|}{$M=5$} \\
\cline { 2 - 14 } \multicolumn{1}{c|}{} & SEL & Mean & StD & $\bar{R}$ & Mean & StD & $\bar{R}$ & Mean & StD & $\bar{R}$ & Mean & StD & $\bar{R}$ \\
\hline \multirow{2}{*}{ WFG1 } & $T U R$ & 1.483 & 12.600 & 1.67 & 1.278 & 9.399 & 1.75 & 1.396 & 4.863 & 1.65 & 2.413 & 3.658 & 1.66 \\
& $R M P$ & 1.532 & 13.366 & 1.33 & 0.751 & 4.996 & 1.25 & 0.984 & 2.229 & 1.35 & 1.763 & 3.676 & 1.34 \\
\hline \multirow{2}{*}{ WFG2 } & TUR & 0.836 & 3.800 & 1.67 & 1.124 & 3.178 & 1.64 & 1.173 & 2.109 & 1.47 & 7.263 & 30.693 & 1.44 \\
& $R M P$ & 0.362 & 2.101 & 1.33 & 0.797 & 2.371 & 1.36 & 1.840 & 4.605 & 1.53 & 4.750 & 10.018 & 1.56 \\
\hline \multirow{2}{*}{ WFG3 } & $T U R$ & 0.276 & 2.480 & 1.51 & 0.148 & 0.966 & 1.58 & 0.069 & 0.085 & 1.53 & 0.181 & 0.204 & 1.54 \\
& $R M P$ & 0.304 & 2.846 & 1.49 & 0.124 & 0.967 & 1.42 & 0.073 & 0.097 & 1.47 & 0.167 & 0.170 & 1.46 \\
\hline \multirow{2}{*}{ WFG4 } & $T U R$ & 2.836 & 28.251 & 1.50 & 0.371 & 2.048 & 1.53 & 0.710 & 1.147 & 1.58 & 2.169 & 5.710 & 1.44 \\
& $R M P$ & 0.265 & 2.597 & 1.50 & 0.196 & 0.946 & 1.47 & 0.831 & 2.402 & 1.42 & 2.153 & 4.565 & 1.56 \\
\hline \multirow{2}{*}{ WFG5 } & $T U R$ & 3.311 & 30.610 & 1.52 & 1.116 & 8.247 & 1.42 & 1.857 & 6.012 & 1.49 & 2.499 & 5.413 & 1.46 \\
& $R M P$ & 2.659 & 24.168 & 1.48 & 1.284 & 9.847 & 1.58 & 1.728 & 6.661 & 1.51 & 3.860 & 15.540 & 1.54 \\
\hline \multirow{2}{*}{ WFG6 } & $T U R$ & 1.129 & 10.237 & 1.61 & 0.911 & 7.205 & 1.43 & 1.213 & 4.240 & 1.54 & 2.376 & 7.831 & 1.51 \\
& $R M P$ & 0.793 & 7.355 & 1.39 & 0.720 & 5.458 & 1.57 & 1.033 & 3.256 & 1.46 & 2.154 & 5.275 & 1.49 \\
\hline \multirow{2}{*}{ WFG7 } & $T U R$ & 0.032 & 0.291 & 1.54 & 0.485 & 3.720 & 1.46 & 0.655 & 2.312 & 1.43 & 2.371 & 6.684 & 1.53 \\
& $R M P$ & 0.004 & 0.011 & 1.46 & 0.189 & 0.683 & 1.54 & 1.025 & 4.547 & 1.57 & 2.711 & 14.009 & 1.47 \\
\hline \multirow{2}{*}{ WFG8 } & $T U R$ & 1.970 & 14.462 & 1.50 & 6.505 & 61.815 & 1.40 & 1.422 & 5.780 & 1.50 & 3.266 & 9.228 & 1.54 \\
& $R M P$ & 1.324 & 11.668 & 1.50 & 0.398 & 1.328 & 1.60 & 1.319 & 5.035 & 1.50 & 4.287 & 14.921 & 1.46 \\
\hline \multirow{2}{*}{ WFG9 } & $T U R$ & 3.899 & 32.327 & 1.52 & 1.005 & 6.168 & 1.49 & 1.676 & 7.136 & 1.46 & 3.330 & 8.487 & 1.46 \\
& $R M P$ & 2.599 & 23.345 & 1.48 & 0.958 & 6.461 & 1.51 & 2.193 & 9.124 & 1.54 & 3.515 & 10.770 & 1.54 \\
\hline
\end{tabular}

Table 4: Average ARSD and respective ranks $\bar{R}$ attained by iMOEA-HA with $S E L \in\{T U R, R M P\}$ and $K=20$, applied to WFG1-9 problems with $M=2-5$ objectives.

\begin{tabular}{|l|c|ccc|ccc|ccc|ccc|}
\cline { 3 - 14 } \multicolumn{1}{c|}{} & \multicolumn{3}{c|}{$M=2$} & \multicolumn{3}{c|}{$M=3$} & \multicolumn{3}{c|}{$M=4$} & \multicolumn{3}{c|}{$M=5$} \\
\cline { 2 - 14 } \multicolumn{1}{c|}{ WFG1 } & SEL & Mean & StD & $\bar{R}$ & Mean & StD & $\bar{R}$ & Mean & StD & $\bar{R}$ & Mean & StD & $\bar{R}$ \\
& TUR & 1.785 & 15.825 & 1.54 & 0.930 & 6.227 & 1.79 & 1.217 & 3.473 & 1.66 & 2.311 & 3.650 & 1.67 \\
& $R M P$ & 1.467 & 12.697 & 1.46 & 0.661 & 4.341 & 1.21 & 0.866 & 2.116 & 1.34 & 1.828 & 3.797 & 1.33 \\
\hline \multirow{2}{*}{ WFG2 } & $T U R$ & 0.844 & 4.093 & 1.63 & 1.026 & 3.093 & 1.53 & 2.136 & 5.430 & 1.55 & 3.820 & 10.285 & 1.47 \\
& $R M P$ & 0.342 & 1.704 & 1.37 & 2.085 & 8.382 & 1.47 & 2.051 & 5.085 & 1.45 & 5.656 & 14.964 & 1.53 \\
\hline \multirow{2}{*}{ WFG3 } & $T U R$ & 0.196 & 1.507 & 1.64 & 0.064 & 0.280 & 1.51 & 0.071 & 0.099 & 1.54 & 0.171 & 0.193 & 1.56 \\
& $R M P$ & 0.084 & 0.634 & 1.36 & 0.087 & 0.491 & 1.49 & 0.061 & 0.073 & 1.46 & 0.163 & 0.170 & 1.44 \\
\hline \multirow{2}{*}{ WFG4 } & $T U R$ & 0.440 & 4.350 & 1.47 & 0.233 & 0.947 & 1.50 & 1.190 & 6.152 & 1.50 & 1.921 & 4.347 & 1.48 \\
& $R M P$ & 0.048 & 0.303 & 1.53 & 0.157 & 0.360 & 1.50 & 1.013 & 4.015 & 1.50 & 1.691 & 3.208 & 1.52 \\
\hline \multirow{2}{*}{ WFG5 } & $T U R$ & 2.645 & 24.168 & 1.56 & 1.097 & 8.465 & 1.51 & 1.478 & 6.297 & 1.51 & 2.639 & 5.601 & 1.43 \\
& $R M P$ & 2.642 & 24.168 & 1.44 & 1.151 & 9.146 & 1.49 & 1.631 & 5.748 & 1.49 & 3.095 & 7.295 & 1.57 \\
\hline \multirow{2}{*}{ WFG6 } & $T U R$ & 1.921 & 17.791 & 1.44 & 1.021 & 8.487 & 1.53 & 1.107 & 4.866 & 1.57 & 1.873 & 3.885 & 1.50 \\
& $R M P$ & 0.567 & 5.150 & 1.56 & 1.024 & 8.976 & 1.47 & 0.897 & 3.901 & 1.43 & 2.194 & 4.950 & 1.50 \\
\hline \multirow{2}{*}{ WFG7 } & $T U R$ & 0.031 & 0.198 & 1.56 & 0.204 & 0.657 & 1.53 & 1.059 & 3.520 & 1.56 & 1.495 & 2.883 & 1.47 \\
& $R M P$ & 0.054 & 0.510 & 1.44 & 0.235 & 0.718 & 1.47 & 0.643 & 1.273 & 1.44 & 1.902 & 6.944 & 1.53 \\
\hline \multirow{2}{*}{ WFG8 } & $T U R$ & 1.356 & 11.647 & 1.59 & 0.389 & 1.263 & 1.53 & 1.221 & 4.822 & 1.48 & 2.913 & 8.542 & 1.60 \\
& $R M P$ & 1.317 & 11.636 & 1.41 & 0.282 & 0.939 & 1.47 & 1.306 & 4.784 & 1.52 & 2.510 & 7.503 & 1.40 \\
\hline \multirow{2}{*}{ WFG9 } & $T U R$ & 3.028 & 24.484 & 1.37 & 1.338 & 11.113 & 1.49 & 1.964 & 8.906 & 1.51 & 4.067 & 15.200 & 1.52 \\
& $R M P$ & 1.708 & 14.249 & 1.63 & 1.355 & 10.864 & 1.51 & 2.282 & 13.293 & 1.49 & 3.444 & 9.938 & 1.48 \\
\hline
\end{tabular}

to 2.11. However, switching from the tournament selection to a restricted mating pool helped handle the bias in WFG1, resulting in the best-attained average ranks ranging from 1.57 to 1.83 . 
Table 5: Average ARSD and respective ranks $\bar{R}$ attained by iMOEA-HA with $K \in\{5,10,15,20\}$, applied to WFG1-9 problems with $M=2-5$ objectives.

\begin{tabular}{|c|c|c|c|c|c|c|c|c|c|c|c|c|c|}
\hline & & \multicolumn{3}{|c|}{$M=2$} & \multicolumn{3}{|c|}{$M=3$} & \multicolumn{3}{|c|}{$M=4$} & \multicolumn{3}{|c|}{$M=5$} \\
\hline & $K$ & Mean & $\mathrm{StD}$ & $\bar{R}$ & lean & StD & $\bar{R}$ & Tere & StD & $\bar{R}$ & Mean & StD & $\bar{R}$ \\
\hline \multirow{4}{*}{ WFG1 } & 5 & 1.780 & 15.422 & 2.90 & 937 & 6.621 & 2.71 & 1.501 & 5.269 & 2.84 & 2.518 & 3.892 & 3.08 \\
\hline & 10 & 1.285 & 11.022 & 2.43 & 0.815 & 5.624 & 2.62 & 0.970 & 2.248 & 2.52 & 2.294 & 3.860 & 2.60 \\
\hline & 15 & 1.532 & 13.366 & 2.36 & 0.751 & 4.996 & 2.44 & 0.984 & 2.229 & 2.37 & 1.763 & 3.676 & 2.23 \\
\hline & 20 & 1.467 & 12.697 & 2.31 & 0.661 & 4.341 & 2.23 & 0.866 & 2.116 & 2.27 & 1.828 & 3.797 & 2.09 \\
\hline \multirow{4}{*}{ WFG2 } & 5 & 0.383 & 2.294 & 2.35 & 2.024 & 8.376 & 2.73 & 2.653 & 6.685 & 2.76 & 6.138 & 16.087 & 2.63 \\
\hline & 10 & 0.451 & 2.828 & 2.53 & 2.040 & 8.961 & 2.66 & 1.853 & 4.484 & 2.56 & 4.449 & 10.297 & 2.55 \\
\hline & 15 & 0.362 & 2.101 & 2.61 & 0.797 & 2.371 & 2.09 & 1.840 & 4.605 & 2.33 & 4.750 & 10.018 & 2.43 \\
\hline & 20 & 0.342 & 1.704 & 2.51 & 2.085 & 8.382 & 2.52 & 2.051 & 5.085 & 2.35 & 5.656 & 14.964 & 2.39 \\
\hline \multirow{4}{*}{ WFG3 } & 5 & 0.601 & 5.700 & 2.21 & 0.157 & 1.036 & 2.77 & 0.106 & 0.156 & 2.69 & 0.190 & 0.177 & 2.73 \\
\hline & 1 & 0.752 & 7.329 & 2.61 & 133 & 1.054 & 2.37 & 0.079 & 0.091 & 2.52 & 0.176 & 0.179 & 2.46 \\
\hline & 15 & 0.304 & 2.846 & 2.73 & 0.124 & 0.967 & 2.36 & 0.073 & 0.097 & 2.36 & 0.167 & 0.170 & 2.51 \\
\hline & 20 & 0.084 & 0.634 & 2.45 & 0.087 & 0.491 & 2.50 & 0.061 & 0.073 & 2.43 & 0.163 & 0.170 & 2.30 \\
\hline \multirow{4}{*}{ WFG4 } & 5 & 0.232 & 1.761 & 2.95 & 0.993 & 8.420 & 2.75 & 1.244 & 2.601 & 2.78 & 2.944 & 5.601 & 2.83 \\
\hline & 10 & 0.047 & 0.408 & 2.48 & 0.353 & 2.100 & 2.72 & 0.985 & 2.016 & 2.49 & 1.835 & 3.587 & 2.38 \\
\hline & 15 & 0.265 & 2.597 & 2.29 & 0.196 & 0.946 & 2.33 & 0.831 & 2.402 & 2.26 & 2.153 & 4.565 & 2.43 \\
\hline & 20 & 0.048 & 0.303 & 2.28 & 0.157 & 0.360 & 2.20 & 1.013 & 4.015 & 2.47 & 1.691 & 3.208 & 2.36 \\
\hline \multirow{4}{*}{ WFG5 } & . & 2.830 & 25.780 & 2.91 & 173 & 18.686 & 2.62 & 1.749 & 6.532 & 2.65 & 3.996 & 13.665 & 2.67 \\
\hline & 10 & 2.940 & 26.517 & 2.45 & 1.146 & 8.456 & 2.53 & 1.911 & 6.489 & 2.62 & 3.461 & 8.332 & 2.59 \\
\hline & 15 & 2.659 & 24.168 & 2.48 & 1.284 & 9.847 & 2.51 & 1.728 & 6.661 & 2.48 & 3.860 & 15.540 & 2.36 \\
\hline & 20 & 2.642 & 24.168 & 2.16 & 1.151 & 9.146 & 2.34 & 1.631 & 5.748 & 2.25 & 3.095 & 7.295 & 2.38 \\
\hline \multirow{4}{*}{ WFG6 } & & 0.918 & 8.501 & 2.64 & 0.878 & 5.719 & 2.91 & 2.750 & 13.900 & 2.81 & 3.047 & 6.989 & 2.89 \\
\hline & 10 & 0.377 & 3.232 & 2.50 & 0.679 & 5.067 & 2.24 & 1.380 & 5.999 & 2.56 & 2.457 & 5.048 & 2.57 \\
\hline & 15 & 0.793 & 7.355 & 2.34 & 0.720 & 5.458 & 2.60 & 1.033 & 3.256 & 2.35 & 2.154 & 5.275 & 2.35 \\
\hline & 20 & 0.567 & 5.150 & 2.52 & 1.024 & 8.976 & 2.25 & 0.897 & 3.901 & 2.28 & 2.194 & 4.950 & 2.19 \\
\hline \multirow{4}{*}{ WFC } & 5 & 0.138 & 1.300 & 2.90 & 0.811 & 6.098 & 2.59 & 1.710 & 6.539 & 2.86 & 3.433 & 8.894 & 2.79 \\
\hline & 10 & 1.139 & 11.349 & 2.42 & 0.227 & 0.827 & 2.50 & 0.927 & 2.410 & 2.51 & 1.940 & 3.492 & 2.65 \\
\hline & 15 & 0.004 & 0.011 & 2.40 & 0.189 & 0.683 & 2.50 & 1.025 & 4.547 & 2.44 & 2.711 & 14.009 & 2.34 \\
\hline & 20 & 0.054 & 0.510 & 2.28 & 0.235 & 0.718 & 2.41 & 0.643 & 1.273 & 2.19 & 1.902 & 6.944 & 2.22 \\
\hline \multirow{4}{*}{ WFG8 } & 5 & 1.394 & 12.027 & 2.65 & 2.962 & 26.051 & 2.61 & 1.454 & 4.949 & 2.63 & 4.278 & 12.458 & 3.07 \\
\hline & 10 & 1.744 & 15.377 & 2.58 & 0.230 & 0.362 & 2.62 & 1.523 & 5.744 & 2.63 & 2.579 & 5.898 & 2.49 \\
\hline & 15 & 1.324 & 11.668 & 2.51 & 0.398 & 1.328 & 2.56 & 1.319 & 5.035 & 2.46 & 4.287 & 14.921 & 2.31 \\
\hline & 20 & 1.317 & 11.636 & 2.26 & 0.282 & 0.939 & 2.21 & 1.306 & 4.784 & 2.28 & 2.510 & 7.503 & 2.13 \\
\hline \multirow{4}{*}{ WFG9 } & - & 1.991 & 16.818 & 3.00 & 1.841 & 13.174 & 2.91 & 2.264 & 9.001 & 2.77 & 4.221 & 12.490 & 2.87 \\
\hline & 10 & 2.529 & 21.645 & 2.53 & 2.184 & 18.882 & 2.46 & 3.255 & 20.156 & 2.48 & 4.194 & 11.928 & 2.63 \\
\hline & 15 & 2.599 & 23.345 & 2.36 & 0.958 & 6.461 & 2.35 & 2.193 & 9.124 & 2.42 & 3.515 & 10.770 & 2.37 \\
\hline & 20 & 1.708 & 14.249 & 2.11 & 1.355 & 10.864 & 2.28 & 2.282 & 13.293 & 2.33 & 3.444 & 9.938 & 2.13 \\
\hline
\end{tabular}


Table 6: Average execution times of EMOSOR and iMOEA-HA with $K \in\{5,10,15,20\}$, applied to WFG1-9 problems with $M=2-$ 5 objectives.

\begin{tabular}{|c|c|c|c|c|c|c|c|c|c|}
\hline & \multirow{3}{*}{ Method } & \multirow{2}{*}{\multicolumn{2}{|c|}{$M=2$}} & \multirow{2}{*}{\multicolumn{2}{|c|}{$M=3$}} & \multirow{2}{*}{\multicolumn{2}{|c|}{$M=4$}} & \multirow{2}{*}{\multicolumn{2}{|c|}{$M=5$}} \\
\hline & & & & & & & & & \\
\hline & & Mean & StD & Mean & StD & Mean & StD & Mean & StD \\
\hline \multirow{5}{*}{ WFG1 } & \multirow{5}{*}{$\begin{array}{c}\text { EMOSOR } \\
\text { iMOEA-HA with } K=5 \\
\text { iMOEA-HA with } K=10 \\
\text { iMOEA-HA with } K=15 \\
\text { iMOEA-HA with } K=20\end{array}$} & 2.185 & 0.247 & 8.67 & 0.153 & 20.313 & 0.81 & 37.508 & 0.223 \\
\hline & & 0.616 & 0.067 & 1.98 & 0.106 & 4.175 & 0.10 & 7.302 & 0.091 \\
\hline & & 0.853 & 0.066 & 2.83 & 0.142 & 5.931 & 0.26 & 9.892 & 0.268 \\
\hline & & 1.151 & 0.087 & 3.99 & 0.159 & 7.845 & 0.30 & 13.227 & 0.231 \\
\hline & & 1.481 & 0.117 & 5.38 & 0.255 & 10.007 & 0.57 & 16.807 & 0.555 \\
\hline \multirow{5}{*}{ WFG2 } & \multirow{5}{*}{$\begin{array}{c}\text { EMOSOR } \\
\text { iMOEA-HA with } K=5 \\
\text { iMOEA-HA with } K=10 \\
\text { iMOEA-HA with } K=15 \\
\text { iMOEA-HA with } K=20\end{array}$} & 2.269 & 0.131 & 8.71 & 0.399 & 19.730 & 0.26 & 46.617 & 16.739 \\
\hline & & 0.467 & 0.069 & 1.82 & 0.085 & 4.064 & 0.13 & 6.437 & 0.566 \\
\hline & & 0.649 & 0.074 & 2.13 & 0.274 & 5.219 & 0.69 & 9.601 & 0.552 \\
\hline & & 0.858 & 0.107 & 3.72 & 0.210 & 7.420 & 0.89 & 11.380 & 1.837 \\
\hline & & 1.049 & 0.150 & 4.41 & 0.637 & 9.806 & 0.77 & 12.965 & 2.548 \\
\hline \multirow{5}{*}{ WFG3 } & \multirow{5}{*}{$\begin{array}{c}\text { EMOSOR } \\
\text { iMOEA-HA with } K=5 \\
\text { iMOEA-HA with } K=10 \\
\text { iMOEA-HA with } K=15 \\
\text { iMOEA-HA with } K=20\end{array}$} & 1.080 & 0.050 & 4.42 & 0.154 & 10.069 & 0.40 & 18.679 & 0.196 \\
\hline & & 0.320 & 0.041 & 1.01 & 0.024 & 2.195 & 0.07 & 3.897 & 0.098 \\
\hline & & 0.413 & 0.043 & 1.45 & 0.032 & 3.118 & 0.08 & 5.326 & 0.062 \\
\hline & & 0.562 & 0.063 & 2.01 & 0.048 & 4.133 & 0.11 & 6.986 & 0.173 \\
\hline & & 0.720 & 0.031 & 2.61 & 0.084 & 5.408 & 0.12 & 8.963 & 0.181 \\
\hline \multirow{5}{*}{ WFG4 } & \multirow{5}{*}{$\begin{array}{c}\text { EMOSOR } \\
\text { iMOEA-HA with } K=5 \\
\text { iMOEA-HA with } K=10 \\
\text { iMOEA-HA with } K=15 \\
\text { iMOEA-HA with } K=20\end{array}$} & 1.094 & 0.056 & 4.34 & 0.173 & 9.904 & 0.10 & 18.820 & 0.159 \\
\hline & & 0.310 & 0.021 & 1.07 & 0.069 & 2.249 & 0.09 & 3.914 & 0.091 \\
\hline & & 0.431 & 0.018 & 1.52 & 0.044 & 3.141 & 0.13 & 5.322 & 0.209 \\
\hline & & 0.591 & 0.063 & 2.05 & 0.076 & 4.146 & 0.13 & 6.872 & 0.214 \\
\hline & & 0.737 & 0.034 & 2.63 & 0.147 & 5.261 & 0.30 & 8.792 & 0.207 \\
\hline \multirow{5}{*}{ WFG5 } & \multirow{5}{*}{$\begin{array}{c}\text { EMOSOR } \\
\text { iMOEA-HA with } K=5 \\
\text { iMOEA-HA with } K=10 \\
\text { iMOEA-HA with } K=15 \\
\text { iMOEA-HA with } K=20\end{array}$} & 1.041 & 0.040 & 4.47 & 0.253 & 9.858 & 0.10 & 18.780 & 0.156 \\
\hline & & 0.337 & 0.037 & 1.06 & 0.054 & 2.294 & 0.05 & 3.858 & 0.081 \\
\hline & & 0.447 & 0.034 & 1.50 & 0.068 & 3.100 & 0.08 & 5.212 & 0.122 \\
\hline & & 0.603 & 0.044 & 2.09 & 0.076 & 4.126 & 0.15 & 6.746 & 0.175 \\
\hline & & 0.775 & 0.068 & 2.69 & 0.148 & 5.161 & 0.34 & 8.683 & 0.220 \\
\hline \multirow{5}{*}{ WFG6 } & \multirow{5}{*}{$\begin{array}{c}\text { EMOSOR } \\
\text { iMOEA-HA with } K=5 \\
\text { iMOEA-HA with } K=10 \\
\text { iMOEA-HA with } K=15 \\
\text { iMOEA-HA with } K=20\end{array}$} & 1.122 & 0.074 & 4.30 & 0.038 & 9.882 & 0.07 & 18.845 & 0.205 \\
\hline & & 0.322 & 0.025 & 1.04 & 0.037 & 2.202 & 0.12 & 3.764 & 0.133 \\
\hline & & 0.430 & 0.013 & 1.47 & 0.076 & 2.973 & 0.16 & 5.180 & 0.154 \\
\hline & & 0.548 & 0.060 & 1.93 & 0.162 & 4.043 & 0.25 & 6.693 & 0.219 \\
\hline & & 0.719 & 0.028 & 2.53 & 0.122 & 5.204 & 0.24 & 8.525 & 0.387 \\
\hline \multirow{5}{*}{ WFG7 } & \multirow{5}{*}{$\begin{array}{c}\text { EMOSOR } \\
\text { iMOEA-HA with } K=5 \\
\text { iMOEA-HA with } K=10 \\
\text { iMOEA-HA with } K=15 \\
\text { iMOEA-HA with } K=20\end{array}$} & 1.047 & 0.033 & 4.30 & 0.134 & 9.896 & 0.12 & 18.807 & 0.119 \\
\hline & & 0.313 & 0.017 & 1.09 & 0.069 & 2.292 & 0.06 & 3.997 & 0.091 \\
\hline & & 0.459 & 0.044 & 1.54 & 0.057 & 3.139 & 0.16 & 5.389 & 0.122 \\
\hline & & 0.582 & 0.018 & 2.08 & 0.057 & 4.199 & 0.20 & 6.971 & 0.137 \\
\hline & & 0.743 & 0.021 & 2.69 & 0.141 & 5.390 & 0.20 & 8.922 & 0.152 \\
\hline & EMOSOR & 1.121 & 0.059 & 4.38 & 0.066 & 10.035 & 0.09 & 19.014 & 0.156 \\
\hline & iMOEA-HA with $K=5$ & 0.399 & 0.153 & 1.11 & 0.092 & 2.313 & 0.09 & 4.141 & 0.154 \\
\hline WFG8 & iMOEA-HA with $K=10$ & 0.471 & 0.046 & 1.58 & 0.105 & 3.239 & 0.16 & 5.647 & 0.159 \\
\hline & iMOEA-HA with $K=15$ & 0.607 & 0.061 & 2.11 & 0.115 & 4.395 & 0.29 & 7.221 & 0.423 \\
\hline & iMOEA-HA with $K=20$ & 0.746 & 0.079 & 2.69 & 0.175 & 5.592 & 0.44 & 9.402 & 0.217 \\
\hline & EMOSOR & 1.152 & 0.039 & 4.44 & 0.085 & 10.221 & 0.14 & 19.293 & 0.182 \\
\hline & iMOEA-HA with $K=5$ & 0.409 & 0.036 & 1.26 & 0.051 & 2.623 & 0.10 & 4.392 & 0.132 \\
\hline WFG9 & iMOEA-HA with $K=10$ & 0.537 & 0.053 & 1.72 & 0.124 & 3.460 & 0.13 & 5.881 & 0.230 \\
\hline & iMOEA-HA with $K=15$ & 0.676 & 0.025 & 2.21 & 0.162 & 4.447 & 0.30 & 7.278 & 0.470 \\
\hline & iMOEA-HA with $K=20$ & 0.819 & 0.089 & 2.90 & 0.120 & 5.571 & 0.43 & 9.034 & 0.547 \\
\hline
\end{tabular}


Table 7: Average ARSD and respective ranks $\bar{R}$ attained by EMOSOR and iMOEA-HA with $S E L \in\{T U R, R M P\}(K=15)$, applied to WFG1 problem with $M=2-5$ objectives.

\begin{tabular}{|c|ccc|ccc|ccc|cc|c|}
\cline { 2 - 13 } \multicolumn{1}{c|}{} & \multicolumn{3}{c|}{$M=2$} & \multicolumn{3}{c|}{$M=3$} & \multicolumn{3}{c|}{$M=4$} & \multicolumn{3}{c|}{$M=5$} \\
\hline Method & Mean & StD & $\bar{R}$ & Mean & StD & $\bar{R}$ & Mean & StD & $\bar{R}$ & Mean & StD & $\bar{R}$ \\
\hline EMOSOR & 1.161 & 9.702 & 1.86 & 2.061 & 17.386 & 2.11 & 1.366 & 5.079 & 2.11 & 2.131 & 3.753 & 1.97 \\
iMOEA-HA with SEL $=$ TUR & 1.483 & 12.600 & 2.31 & 1.278 & 9.399 & 2.32 & 1.396 & 4.863 & 2.14 & 2.413 & 3.658 & 2.23 \\
iMOEA-HA with SEL $=$ RMP & 1.532 & 13.366 & 1.83 & 0.751 & 4.996 & 1.57 & 0.984 & 2.229 & 1.75 & 1.763 & 3.676 & 1.80 \\
\hline
\end{tabular}

